www.jmscr.igmpublication.org

Impact Factor 5.84

Index Copernicus Value: 71.58

ISSN (e)-2347-176x ISSN (p) 2455-0450

crossref DOI: _https://dx.doi.org/10.18535/jmscr/v5i9.128

\title{
Sexual Dysfunctions and Affecting Factors in Middle Aged Indian Females
}

\author{
Author \\ Goyal Ritu \\ Assistant Professor, Department of Obstetrics and Gynaecology \\ AIMSR, Bathinda, Punjab, India
}

\begin{abstract}
Female sexual disorders are complex, diverse and associated with multiple factors. They are highly prevelant in our society but often not recognized, diagnosed and managed. There is a paucity of studies evaluating this condition in Indian perspective. So a study was conducted in department of obstetrics and gynaecology in Adesh Institute of Medical Sciences And Research on 100 patients of middle age group attending OPD clinic. Mean age of patients with sexual dysfunction was 47_+ 7 yrs. $65 \%$ patients reported sexual dysfunction. Females living in joint families, with sedentary life style, married for $>20 y e a r s$ and housewives had more problems related to FSDs. But out of all females with problem only $17 \%$ consulted doctor for the problem. It gives us interpretation that in our set up this issue is still limited by social and cultural issues which needs to be assessed and addressed properly.

Key Words: sexual dysfunction, menopause, dysparunia, Arousal.
\end{abstract}

\section{Introduction}

Sexuality is a part of individual 's personality. This is an important aspect of human life but largely ignored especially in developing countries. It is influenced by various factors as gynaecological disorders, medical diseases as cardiovascular diseases, hypertension and diabetes mellitus are more common in this age group. Long use of certain drugs, psychological state of the person and social beliefs also have considerable impact on sexual function ${ }^{(1)}$. Most of Indian women consider intercourse as a way to reproduce only and feel themselves sexually retired after menopause $^{(2,3,4)}$. They feel embarrassed to talk about their sexual dysfunction due to cultural taboos. In 2001 Berman et al found that with increasing age female sexual disorders (FSD) increases $^{(5)}$. It may be due to decline in hormones leading to urogenital atrophy ${ }^{(6)}$. Also due to deceased physical activity menopausal symptoms become more intense like mood, depression, anxiety, decreased sleep and musculoskeletal disorders leading to diminished quality of life $(7,8,9)$. Sometimes these FSDs are due to sexual pain which may be caused by PID, deep infiltrating lesions of pelvis or endometriosis which can be treated accordingly ${ }^{(10)}$.

\section{Materials and Methods}

This is a cross sectional study conducted in department of obstetrics and gynaecology in AIMSR Bathinda. 100 fertile females who were in age group 35-55yrs attending obstetrics and gynaecology OPD were included in study. A questionnaire was designed to obtain information on demographic characters and sexual functioning 
of women in perimenopausa 1 age group. To assess the desire, arousal, orgasm, sexual pain, their satisfaction and spouse satisfaction questions were framed. Each question was scored from 0 (no problem) to 4 (worst problem). Total score was from 0-48 and based on total score subjects were classified into three groups. 0-24 normal, 2536 borderline, 37-48 severe.

Illiterate women were questioned in person. The females with known diseases that have effect on sexual function as thyroid disease, heart or lung disease, renal disease, diabetes, genitalia injury, or who were on medications like antidepressants, antihypertensive, opiods or females with sexual dysfunction in spouse were excluded from study.

\section{Observations}

In our study, the mean age of females was $47++7$ yrs and mean duration of marriage was 21_+3yrs. $65 \%$ of subjects had sexual dysfunctions. It was more prevalent in $>50 y$ rs age group.

\section{Table 1}

\begin{tabular}{|l|c|c|}
\hline $\begin{array}{l}\text { Age Group } \\
\text { (years) }\end{array}$ & FSD present $(\mathrm{n}=65)$ & $\begin{array}{c}\text { FSD absent } \\
(\mathrm{n}=35)\end{array}$ \\
\hline$<35$ & $8(12.3 \%)$ & $12(34.2 \%)$ \\
\hline $35-40$ & $10(15.3 \%)$ & $9(25.7 \%)$ \\
\hline $45-50$ & $15(20 \%)$ & $7(20 \%)$ \\
\hline $50-55$ & $18(27.6 \%)$ & $4(11.4 \%)$ \\
\hline$>55$ & $14(21.5 \%)$ & $3(8.5 \%)$ \\
\hline
\end{tabular}

Various demographic factors as education, occupation, socioeconomic status, and duration of marriage were studied with respect to prevalence of FSD. Majority of the females (69.2\%) with sexual dysfunction were housewives. Majority of affected females had financial problems. All of them were living with single partner. FSD was more common in females who were less educated (44.6\%). It was more prevalent in females with duration of marriage >20yrs (47.6\%). The prevalence of dysfunction was more in females living in joint families $(61.5 \%)$ that seemed to be due to lack of privacy and social taboos that age of sexual activity is now gone. $26 \%$ females reported lesser frequency of sex as their children are grown up now and due to some other social taboos regarding sex. A major cause in $18 \%$ of females in
$<45 y$ rs age group was marital dissatisfaction, marital discord including alcohol abuse by husband, extramarital relationship or in some cases physical abuse.

\section{Table 2}

\begin{tabular}{|l|l|}
\hline Demographic variables & FSD present $(\mathrm{n}=65)$ \\
\hline Education & \\
\hline Illiterate & $17(26.1 \%)$ \\
\hline Primary education & $29(44.6 \%)$ \\
\hline Higher education & $19(29.2 \%)$ \\
\hline Occupation & \\
\hline Housewife & $45(69.2 \%)$ \\
\hline Working & $20(30.7 \%)$ \\
\hline Family & \\
\hline Joint & $40(61.5 \%)$ \\
\hline Nuclear & $25(38.4 \%)$ \\
\hline Duration of marriage & \\
\hline$<10$ & $7(10.7 \%)$ \\
\hline $10-20$ & $27(41.5 \%)$ \\
\hline$>20$ & $31(47.6 \%)$ \\
\hline & \\
\hline
\end{tabular}

Sexual function is also greatly affected by physical activity level of females. Sedentary life style individuals have higher prevalence of sexual dysfunctions than physically active females (44.4\%).

\section{Table 3}

\begin{tabular}{|l|c|}
\hline Physical activity level & Sexual dysfunction \\
\hline Active & $13(20 \%)$ \\
\hline Moderately active & $23(35.3 \%)$ \\
\hline Sedentary & $29(44.4 \%)$ \\
\hline
\end{tabular}

In our study $65 \%$ of subjects had sexual dysfunction in different phases. More than half had severe dysfunction based on scoring system, one third were facing borderline dysfunction. Desire and arousal dysfunction are predominant in females. A significant proportion of females mentioned reduced interest in sex. Only 35\% had satisfaction with their sexual life. But out of those affected only $17 \%$ consulted doctor for such problems. $23 \%$ females reported that they now rarely have any sexual activity and had abstinence for varying periods of time. $70 \%$ report decrease in frequency of intercourse after menopause. $69 \%$ reported painful intercourse. $62 \%$ mentioned lack of sexual satisfaction. Figure 1 
Table 4

\begin{tabular}{|l|c|}
\hline Type of dysfunction & FSD present $(\mathrm{n}=65)$ \\
\hline Desire & $78 \%$ \\
\hline Arousal & $82 \%$ \\
\hline Orgasm & $70 \%$ \\
\hline Satisfaction & $62 \%$ \\
\hline Pain & $69 \%$ \\
\hline
\end{tabular}

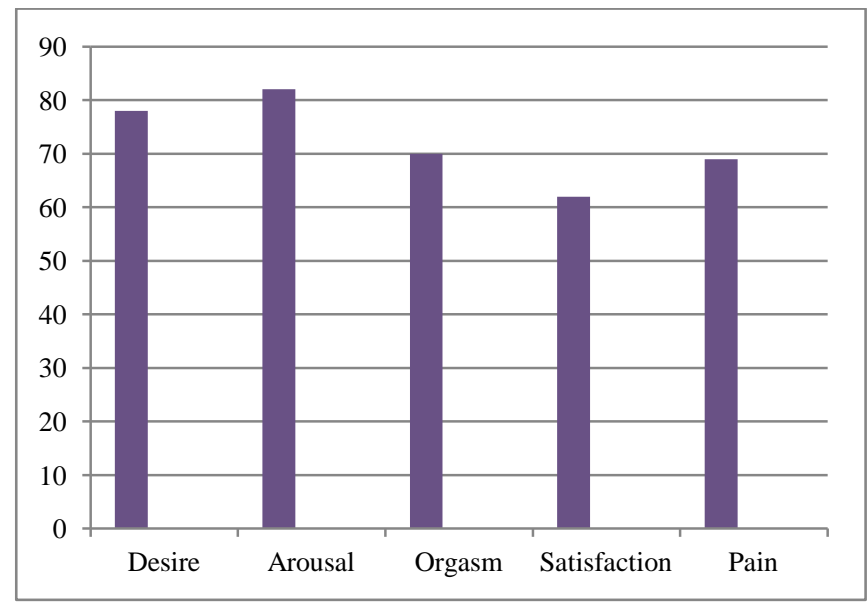

\section{Discussion}

In this study $72.3 \%$ of subjects reported a decrease in sexual activity after menopause. In 2001 Berman et al reported that with increase in age, FSD also increases ${ }^{(5)}$. This incidence is somewhat similar to Park's study in Korea ${ }^{(11)}$ and Dhillon's study in Malaysia ${ }^{(12)}$. In our study $65 \%$ of total females had sexual dysfunction but according to Armaan et al the frequency in Isfahan is $72.4 \%$ (13). In Addis's study the frequency was $45 \%$ in middle aged and elderly women ${ }^{(14)}$. In Bonnie's study it was $20 \%{ }^{(15)}$. In our study higher frequency may be due to sociocultural differences. Women's education and socioeconomic status do play a role in her outlook toward sexual life. In a study by Fajewonyomi et al ${ }^{(16)}$ they found that FSD was more prevalent in women with higher education (43.4\%) as compared to women with no formal education (7.3\%). In our study also FSD in illiterate women was $26.1 \%$ and in women with somewhat formal education it was $72.9 \%$. This variation may be due to the fact that women who are educated are more frank in discussing their sexual problem with their spouse and have more awareness about the issue ${ }^{(17)}$. Similarly FSD is more prevalent in women with upper socioeconomic status $45.4 \%$ than in lower socioeconomic status $19.9 \%$. The duration of marriage has a negative impact on sexual life. FSD was present in $47.6 \%$ of females with duration of marriage $>20$ years. It may be supported by theory that with increase in duration of marriage, there is decrease in frequency of intercourse leading to satisfaction disorder ${ }^{(18)}$.

Physically inactive or females in sedentary life style suffer more from FSD (44.4\%) than physically active women (20\%). Dabrowska et al reported an association between higher level of general physical activity and better sexual dysfunction in Polish women ${ }^{(7)}$. It is thought that physical activity increases the synaptic transmission of monoamines which supposedly functions in the same manner as antidepressants (19). Similarly Li et al also reported less sexual dysfunction especially vaginal dryness and decreased sexual desire in moderately or very active women $^{(8)}$.

$78 \%$ women reported either an absent or decreased sexual desire that is same as Kaboody study $^{(20)}$ which was done in 2002 in Kermanshah (70\%). Hayes et al found the proportion of European females with low desire increased from $11 \%$ in $20-29 y$ rs to $53 \%$ in females aged $61-70 y r s$ (21). Women with desire disorders are difficult to treat. Use of testosterone and estrogen replacement therapy has shown good results in these patients ${ }^{(22)}$.

In our study, sexual pain disorder was reported by $69 \%$ and it is higher than in other studies. Sexual pain disorder can be due to dysparunia or vaginismus. In Australia it is 12\%, Taiwan 32\% (23). Dysparunia is usually due to decrease in vaginal lubrication or menopausal atrophic changes. $82 \%$ of women stated vaginal dryness after menopause. Vaginal dryness may be due to effect of age and hypoandrogenism ${ }^{(24)}$. These can be treated depending on the underlying cause.

Arousal problem was in $82 \%$ and it was similar to another study in Iran that was done by Arman et al showing it to be $75.3 \%{ }^{(13)}$. Arousal disorders can be treated by encouraging to increase foreplay duration $^{(1)}$. Orgasmic problems were in $70 \%$ and 
Nicolosi et al reported orgasm problem to be in $16 \%{ }^{(25)}$.

$52.5 \%$ subjects mentioned that sexual problems had an effect on their relationship with their husbands, similar to Nicolosi et al who found that $76 \%$ women agreed that satisfactory sex is essential to maintain relationships ${ }^{(25)}$. Most of the females are passive partners in their sexual life. They need to be motivated for intercourse which is largely influenced by the status of their interpersonal conflicts and intimacy ${ }^{(16)}$. Presence of stress, depression, anxiety, and conflicts can have negative impact on their personal as well as sexual life. So it is important to identify all compounding factors significantly associated with presence of sexual dysfunction as age, menopause, marital discord, history of alcohol and physical abuse, lack of privacy at home, financial problems, multiple $>3$ pregnancies, while dealing with FSDs. Literacy and good physical activity are positive factors.

There is a dearth of information about female sexual dysfunctions in India. The FSFI score used to study this dysfunction has to be individualised as it is standardized in west so reports very high score when applied on Indian population ${ }^{(26,27,28)}$. In study of our country we have to take into account our cultures, social structure and status of women here and give due consideration to these factors. There is a belief here that sex is only for reproduction and it tells us the role of social and cultural influences on sexual behaviour. $70 \%$ of females had first information of sex from their husbands. It means the culture here doesn't allow discussion about sex and there is lack of sex education in adolescents or adults. People feel embarrassed to talk about sex openly with anybody else except their partners. Sex clinics giving correct authentic information and guidance are also less. We usually see many men but very few women seeking help for sexual problems in hospital settings. So females report dissatisfaction in their married life in concern to emotional issues rather than talking about sexual issues. That is why large number of females reported that sex was not a priority in their lives and considered that reduced sex interest is only a natural phenomenon due to ageing. So dysfunctions per se without studying other significant personal and social problems cannot be reported. FSFI fails to elicit all these associated problems so cannot recognize the real problem of FSD. That is why we should assess a history of not just the specific problem but also assess personal, interpersonal problems, marital, social and cultural issues ${ }^{(29)}$.

\section{Conclusion}

This data shows that poor sexual function is found in perimenopausal females. The women in our society avoid showing their sexual emotions because of lack of knowledge and traditional beliefs. So promotion of quality of life in this age group is an important issue. It needs more studies as these are largely ignored aspects of human life. Despite the apparent simplicity of current definition and criteria for FSD their use in clinical practice needs careful evaluation. Otherwise there are chances of diagnosing more false positive cases. So we need to enquire about sexual difficulties taking into consideration her personal, culture and social aspect. There is need to establish the impact of FSD on personal and social life and the resulting distress. We should exclude any medical, psychiatric cause and use of any drugs like antidepressants, antihypertensives, antipstchotics or antihistaminics before labelling the dysfunction. Educative and supportive approach to address the problem in wholesome considering all the factors is must. Medication based strategy for FSD should be used as a last resort.

Health and Sex education is must in higher schools to give useful and important information to youngsters. This will help to curb increasing myths and beliefs about sex, allay anxiety among people and most important help them to save from unscrupulous healthcare professionals. So understanding FSD in wholesome needs time, skill and sensitivity towards social and cultural values. 


\section{References}

1. Philips NA. Female sexual dysfunction: Evaluation and treatment. Am Fam Phsician 2000; 62:127-36,141-2.

2. PeateT. Sexuality and sexual health promotion for the older person. Br J Nurs 2004; 13: 188-93.

3. Stotland NL. Menopause : Social expectations women's realities. Arch Womens Ment Health 2002; 5:5-8

4. Young M, Denny G, Young T, Luquis R. Sexual satisfaction among married women age 50 and older. Psychol Rep 2000; 86:1107-22

5. Berman LA, Berman JR, Chhabra S, Goldstein I. Novel approaches to female sexual dysfunction . Expert Opin Investig Drugs 2001;10:85-95.

6. Sarrel PM. Effects of hormone replacement therapy on sexual psychophysiology and behaviour in postmenopause. J Womens Health Gend Based Med 2000;9 Suppl 1: S25-32.

7. Dabrowska J, Skrzypulec V, Drosdzol A. The influence of physical activity level on climactric symptoms in menopausal women. Climactric 2010; 13:355-61

8. Li S, Holm K, Gulanick M, Lanuza D, Penckofer S. The relationship between physical activity and perimenopause. Health Care Women Int 1999; 20:163-78.

9. Llanzea P, Arnott B,Garcia - Portilla MP, Chedraui P, Perez Lopez FR. Sexual function assessment in postmenopausal women with the 14-item changes in sexual functioning questinnnaire. J Sex Med 2011; 8:2144-51

10. ACOG Technical Bulletin. Sexual Dysfunction. No.211. Washington, D.C.: American College of Obstetricians and gynaecologists; September, 1995.

11. Park Y J, Kim HS, Chang SO, Kang HC,Chun SH. Sexuality and related factors of postmenopausal Korean women.
Taehan Kanho Hakhoe Chi 2003; 33:45763

12. Dhillon HK, Singh HJ, Ghaffer NA. Sexual function in menopausal women in Kelantan, Malaysia. Maturitas 2005; 52:256-63

13. Armaan S, Fahami F, Hassan Zahraee R. Comparison of sexual dysfunction before and after menopause among women. A.M.U. J 2005; 8:1-7.

14. Addis IB,Van Den Eeden SK, Wassel-Fyr CL, Vittinghoff E, Brown JS, Thom DH. Sexual activity and function in middle aged and older women. Obstet Gynaecol 2006; 107: 755-64.

15. Bonnie R. Sexual dusfunction (Sex,drugs and women's issues). Prim Care Update Ob Gyns 1999;6:61-5

16. Fajewonoyomi BA, Oriji EO, Adeyemo AO. Sexual dysfunction among female patients of reproductive age in a hospital setting in Nigeria. J Health Popul Nutr 2007; 25: 101-6.

17. Afonja S. Women ,power and authority in traditional Yoruba society. In: Dude L, Leacock E, Ardener S, editors. Visibility and Power. South Hadley, MA: Bergin and Garvey ; 1986. P.136-57.

18. Amidu N, Owiredu WK, Gyasi - Sarpong CK, Woode E, Quaye L. Sexual dysfunction among married couples living in Kumasi metropolis, Ghana. BMC Urol 2011;11:3.

19. Miles L. Physical activity and health. Nutr Bull. 2007; 32(4): 314-63.

20. Kabody M. Knowledge and practice of women above 35 yrs towards menopause. A.M.U.J.2002; 2:101-6. (In Persian)

21. Hayes RD, Dennerstein L, Benett CM , Koochaki PE, Leiblum SR, Graziottin A. Relationship between hypoactive sexual desire disorder and ageing. Fertil Steril 2007; 87: 107-12

22. Gelfand MM, Witta B. Androgen and estrogen -androgen hormone replacement 
therapy: A review of the safety literature, 1941-1996. Clin Ther 1997 ;19:383-404.

23. Pan HA, Wu MH, Hsu CC, Yao BL, Huang KE. The perception of menopause among women in Taiwan. Maturitas 2002; 41:269-74.

24. Gorodeski GI . Vaginal cervical epithelium permeability decreases after menopause. Fertil Steril 2001; 76:753-61.

25. Nicolosi A,Laumann EO, Glasser DB, Moreira EDJr, Paik A, Gingell C. Sexual behaviour and sexual dysfunctions after age 40: The global study of sexual attitudes and behaviours. Urology 2004; 64: 991-7.

26. Singh J,Tharyan P, Kekre NS, Singh G, Gopalakrishnan G. Prevelance and risk factors for female sexual dysfunction in women attending a medical clinic in south India.J Postgrad Med 2009;55:113-20

27. Kulhara P,Avasthi A. Sexual dysfunction on Indian subcontinent. Int Rev Psychiatry $1995 ; 7: 231-9$

28. Avasthi A, Kaur R, Prakash O, Banerjee A, Kumar L, Kulhara P.Sexual behaviour of married young women: Apreliminary study from north India. Indian $\mathbf{J}$ Community Med 2008;33:163-7

29. Varghese KM , Bansal R, Kekre AN, Jacob KS. Validation of the female sexual function index in Southern India. Natl Med J India 2013;26:57. 